\title{
Estructura Factorial y Confiabilidad del Cuestionario de Motivación y Estrategias de Aprendizaje, MSLQ, en Estudiantes Universitarios Chilenos de Primer Año
}

\author{
Factorial Structure and Reliability of the Motivation and Strategies Learning \\ Questionnaire, MSLQ, in Chilean First Year University Students
}

\author{
Bárbara Inzunza ${ }^{1}$, Cristhian Pérez ${ }^{2}$, Carolina Márquez $^{3}$, Liliana Ortiz $^{4}$, Sylvain Marcellini ${ }^{5}$ y \\ Soledad Duk ${ }^{6}$
}

\begin{abstract}
Resumen
El presente estudio analiza la estructura factorial y confiabilidad del cuestionario de Motivación y estrategias de aprendizaje (MSLQ) en una muestra de 409 estudiantes universitarios con edad promedio de 19.08 años, quienes respondieron dicho cuestionario previo consentimiento informado. La estructura factorial se analizó a través de un análisis factorial exploratorio. Los resultados revelan la formación de nueve factores, tres corresponderían a la escala de motivación y seis a la escala de estrategias de aprendizaje. El MSLQ muestra ser un instrumento confiable de medición de la motivación y las estrategias de aprendizaje, aplicable en contexto de investigación, sin embargo es necesario realizar nuevas investigaciones que permitan indagar respecto de las discrepancias con el modelo factorial propuesto por sus autores.
\end{abstract}

Palabras clave: motivación, estrategias de aprendizaje, estudiantes universitarios, Chile

\begin{abstract}
This study analyzes the factorial structure and reliability of the motivation and learning strategies questionnaire (MSLQ) in a sample of 409 university students between with an average age of 19.08. An Exploratory Factor Analysis was carried out using the Principal Axis Extracting method (AEP). The results identified 9 factors, three fitting the scale of motivation and six fitting the scale of learning strategies. The MSLQ proves to be a reliable instrument for measuring motivation and learning strategies applicable in the research context, however further research is needed to investigate the discrepancies with the factor model proposed by their authors.
\end{abstract}

Keywords: motivation, learning strategies, university students, Chile

\footnotetext{
1 Magíster en Docencia para la Educación Superior. Universidad de Concepción. Profesor asistente. Barrio Universitario s/n. Concepción. Chile. Tel.:+56412203800. Correo: binzunza@udec.cl

${ }^{2}$ Magíster en Psicología con mención Psicología Educativa. Universidad de Concepción. Profesor asociado. Barrio Universitario s/n. Concepción. Chile. Tel.: +56412661029. Correo: cperezv@udec.cl

${ }^{3}$ Magíster en Educación Médica para Ciencias de la Salud. Universidad de Concepción. Profesor asistente. Barrio Universitario s/n. Concepción. Chile. Tel.: +56412204927. Correo: cmarquezu@udec.cl

4 Magíster en Educación Superior con mención Pedagogía Universitaria. Universidad de Concepción. Profesor asociado. Barrio Universitario s/n. Concepción. Chile. Tel.: +56412207083. Correo: lilianaortiz@udec.cl

${ }^{5}$ Doctor en Ciencias Biológicas. Profesor asociado. Barrio Universitario s/n. Concepción. Chile. Tel.: +56412203295 . Correo: smarcellini@udec.cl

${ }^{6}$ Profesor en Biología y Ciencias. Profesor asistente. Barrio Universitario s/n. Concepción. Chile. Tel.: +56412203810 .

Correo:sduk@udec.cl
} 


\section{Introducción}

El sistema de educación superior en Chile ha experimentado cambios importantes en cuanto al número de estudiantes que cada año acceden a ella. De acuerdo a cifras del Servicio de Información de Educación Superior (SIES) del Ministerio de Educación de ese país, en el año 2015 el número de matriculados en programas de pregrado incrementó un $14.8 \%$ en los últimos cinco años. Pese a esto, de acuerdo a estimaciones recientes del SIES (2015), en su último informe de retención (cohorte 2010 a 2014) en promedio, 3 de cada 10 estudiantes dejan su carrera durante su primer año de estudios, ya sea por motivos vocacionales, económicos u otros. En términos de género, consistentemente las mujeres muestran una tasa de retención de primer año más alta que los hombres $(73.1 \%$ versus $67.4 \%$, para la cohorte 2014).

Conocer los factores que influyen en la retención de los estudiantes y los motivos que inciden en el abandono temprano de sus estudios es uno de los grandes desafíos que enfrenta el campo de la investigación en educación superior (Díaz, 2008).

Si bien durante algunos años el centro de atención de las investigaciones sobre el aprendizaje estuvo dirigido prioritariamente a la vertiente cognitiva del mismo; en la actualidad existe un consenso generalizado en subrayar una esencial interrelación entre lo cognitivo y lo motivacional (Núñez, 2009; Rinaudo, Chiecher, \& Donolo, 2003). Lo anterior se remonta alrededor de los años ' 60 , luego de la revolución cognitiva, donde surgen nuevas líneas de investigación que enfocan su interés en la importancia de los procesos cognitivos y la motivación en el proceso de aprendizaje.

Estas dos líneas de investigación, que avanzaban por caminos diferentes, confluyen para conjugar ambos componentes en propuestas integradoras sobre cómo tienen lugar los aprendizajes (Cardozo, 2008). Desde los años ochenta se han llevado a cabo numerosos estudios que han mostrado las relaciones e interacciones entre la motivación, las estrategias de aprendizaje y el rendimiento de los estudiantes de diferentes niveles académicos (Pintrich \& De Groot, 1990; Pintrich \& García, 1991; Pintrich, Smith, García,
\& Mckeachie, 1991; Pintrich, Smith, García, \& Mckeachie, 1993; Roces et al., 1999).

Uno de los instrumento ampliamente utilizado con este objetivo es el Cuestionario de Motivación y Estrategias para el Aprendizaje, en inglés, Motivated Strategies Learning Questionnaire (MSLQ) de Pintrich et al. (1991). Éste es una medida diseñada para evaluar la cognición y motivación de tareas específicas, así como las estrategias de aprendizaje utilizadas por los estudiantes para comprometerse con la tarea.

El desarrollo del MSLQ implicó tres grandes recopilaciones de datos en los años 1986, 1987 y 1988 antes de llegar a la versión de 1991, considerada como la versión definitiva del instrumento. Las recopilaciones de 1986, 1987 y 1988 permitieron analizar los datos, reescribir los ítems y refinar el modelo conceptual en el cual subyace el MSLQ (Duncan \& Mckeachie, 2005).

Según la propuesta de Pintrich et al. (1991), el MSLQ se divide en dos secciones una, de estrategias de aprendizajes y otra de motivación. La sección de estrategias de aprendizaje del instrumento se divide en una sección cognitivometacognitiva, que incluye ensayos, elaboración, organización, pensamiento crítico y la autorregulación metacognitiva, y una sección de gestión de recursos, que incluye comportamientos tales como gestión del tiempo y el ambiente de estudio, la gestión del esfuerzo, el aprendizaje por pares y la búsqueda de ayuda. Por su parte, la sección de motivación contiene las escalas que de valoración, expectativas, y afecto.

Previo al MSLQ, gran parte de la investigación sobre el aprendizaje del estudiante universitario se concentraba en las diferencias individuales o estilos de aprendizaje, cuyos vínculos con el comportamiento real de estudio o el procesamiento cognitivo de los estudiantes no eran del todo claros. Además, los inventarios de habilidades de estudio utilizados a la hora de medir el aprendizaje de los estudiantes de la universidad eran criticados por ser ateóricos (Duncan \& Mckeachie, 2005). En cambio, el MSLQ fue desarrollado usando una mirada socialcognitiva de la motivación y de las estrategias aprendizaje, considerando al estudiante como un activo procesador de información cuyas creencias y conocimientos son importantes mediadores de la incorporación de la instrucción (Pintrich et al., 
1993). El marco teórico social-cognitivo en el que se fundó, asume que la motivación y las estrategias de aprendizaje no son rasgos del alumno, sino que la motivación es dinámica y ligada al contexto y que las estrategias de aprendizaje pueden ser aprendidas y puestas bajo el control del estudiante. Es decir, la motivación de los estudiantes varía para los distintos cursos y el uso de estrategias podría variar también dependiendo de la naturaleza de las tareas académicas (Duncan \& Mckeachie, 2005).

Entre las investigaciones realizadas por Pintrich y sus colaboradores donde se ha usado el MSLQ se incluyen estudios que analizan las relaciones entre los componentes del modelo y el rendimiento académico (Pintrich, 1986; Pintrich, 1989; Pintrich \& De Groot, 1990; Pintrich \& García, 1991; Pintrich et al., 1991; Pintrich et al., 1993). Diversos estudios señalan que las subescalas muestran prometedora validez predictiva del rendimiento académico (Kitsantas, Winsler \& Huie, 2008; Khatib, 2010; Pintrich et al., 1993; Rinaudo, De la Barrera, \& Danolo, 2006; Sachs, Law, Chan, \& Rao, 2001).

El año 2011, Credé y Phillips realizaron una revisión meta-analítica del MSLQ basados en 2.158 correlaciones a partir de 67 muestras independientes y $19.900 \quad$ estudiantes universitarios. Sus resultados apoyan ampliamente la estructura teórica del MSLQ y sugieren que es una medida razonablemente fiable. Por otra parte, el patrón de las relaciones entre los constructos evaluados por el MSLQ apoya la idea de que el efecto de las motivaciones en el rendimiento académico está mediado por las estrategias en la forma sugerida por los autores del MLSQ.

En términos psicométricos, investigaciones realizadas por Pintrich y sus colaboradores (1993) demostraron que la consistencia interna del MSLQ fue relativamente buena, y la mayoría de los alfas de Cronbach de las escalas individuales eran aceptables (entre .52 y .93). El MSLQ ha sido traducido a más de 20 idiomas diferentes y se ha sometido a una evaluación formal de validez y fiabilidad en varios otros idiomas como el portugués, español y chino (Saks, Leijen, Edovald \& Õun, 2015) constituyéndose en un instrumento ampliamente utilizado por investigadores e instructores en todo el mundo (Duncan \& Mckeachie, 2005).
En cuanto a versiones disponibles en castellano se encuentra la versión de Roces, Tourón y González Torres (1995), quienes tradujeron y adaptaron el MSLQ a la población universitaria española, el cual se conoce con el nombre de CEAM (cuestionario de Estrategias de aprendizaje y motivación). Se refiere a la motivación y estrategias de aprendizaje que utiliza el alumno en el conjunto de las asignaturas de un curso académico, a diferencia del instrumento original que se refería a una asignatura concreta. Por otra parte, en el estudio de Martínez y Galán (2000) se estimó la consistencia interna del MSLQ en una muestra de estudiantes universitarios españoles, utilizando una versión modificada del original que se compone de 25 ítems de la sección de motivación y 20 de la sección de estrategias de aprendizaje. Se descartaron ítems que carecían de interés para el estudio, de difícil traducción o no adecuados a las características de la muestra y la asignatura. En el estudio de Cardozo (2008) se analizó la consistencia del MSLQ y su relación con el rendimiento académico en estudiantes universitarios venezolanos, utilizando la versión castellana validada por Roces, Tourón y Gonzáles (1995) y Castañeda (1997), sometida a validación por un grupo de expertos con dominio inglés. Finalmente el estudio de Ramírez, CantoRodríguez, Bueno \& Echazarreta (2013) tradujeron y adaptaron el MSLQ a la población universitaria mexicana, sin embargo fue aplicado en un contexto universitario generalizado, considerando estudiantes de facultades, titulaciones y cursos diferentes.

Teniendo en cuenta la importancia de la motivación y de las estrategias de aprendizaje en el proceso de aprendizaje y en ausencia de instrumentos con evidencia de validez $y$ confiabilidad para medirlo en el contexto de Chile, el objetivo de la presente investigación fue explorar las propiedades psicométricas del MSLQ en estudiantes universitarios en una asignatura concreta, específicamente su validez a partir de su estructura factorial, y su confiabilidad, medida a través de su consistencia interna (Martínez, Hernández, \& Hernández, 2006). 


\section{Metodología}

El presente estudio cuantitativo y de carácter psicométrico, se desarrolló mediante un diseño no experimental de corte transversal, a través de encuestas.

\section{Participantes}

La muestra, elegida mediante muestreo no probabilístico consecutivo, estuvo constituida por 409 sujetos de cinco carreras de pregrado diferentes que cursaron la asignatura de biología celular para primer año, de los cuales 95 (23.23\%) eran hombres con una edad que oscilaba entre 17 y 29 años $(M=19.45 ; D . E=1.96)$ y $314(76.77 \%)$ mujeres, con una edad que oscilaba entre 17 y 30 años $(M=18.96 ; \quad D . E=1.72)$. Los estudiantes pertenecían a cinco programas de pregrado diferentes, siendo 54 (13.20\%) de Fonoaudiología, 35 de Ingeniería Ambiental (8.56\%), 136 de Nutrición y dietética (33.25\%), 109 de Obstetricia y puericultura $(26.65 \%)$ y 75 de Tecnología médica $(18.34 \%)$.

\section{Instrumento}

Los participantes respondieron la adaptación al castellano de la versión original y autorizada del Motivated Strategies Learning Questionnaire (MSLQ) diseñado por Pintrich y colaboradores (1991), solicitada a la Universidad de Michigan. El MSLQ es un cuestionario de administración colectiva que consta de 81 ítems, con dos secciones: una referida a la motivación y otra al uso de estrategias de aprendizaje. La sección de motivación está integrada por 31 ítems que conforman seis escalas relativas a distintos aspectos motivacionales de los estudiantes: Metas de orientación intrínseca, Metas de orientación extrínseca, Valoración de la Tarea, Creencias de Autoeficacia, Creencias de Control del Aprendizaje y Ansiedad.

La segunda sección referida al uso de estrategias de aprendizaje, está compuesta por 50 ítems que, de acuerdo a Pintrich, apuntarían a nueve dimensiones: Uso de Estrategias de Repaso, Elaboración, Organización, Pensamiento Crítico, Autorregulación Metacognitiva, Manejo del Tiempo, Ambiente de Estudio, Regulación del Esfuerzo, Aprendizaje con Pares y Búsqueda de Ayuda.
Cada uno de estos ítems consiste en una afirmación ante la cual el estudiante debe responder según el grado de identificación con lo dicho, eligiendo entre una de siete alternativas en una escala de Likert, donde 1 indica poco acuerdo y el 7 indica gran acuerdo con la proposición.

\section{Procedimiento}

Tal y como se indica en los antecedentes previos, las versiones disponibles en castellano del MSLQ constituyen modificaciones del instrumento por lo cual se decidió a realizar una traducción propia desde la versión original del MSQL. La traducción y adaptación al castellano fue realizada por un equipo de tres profesionales bilingües con experiencia en docencia e investigación universitaria.

Una vez finalizada la traducción se exploró la validez de contenido de los ítems, para lo cual se sometió el cuestionario a un juicio de expertos que evaluaron la adecuación, redacción y fidelidad respecto de los ítems en el instrumento original. Este grupo de expertos estuvo conformado por tres docentes universitarios con experiencia en investigación en educación superior.

Se invitó a participar en el estudio a alumnos de primer año de diversas carreras de una universidad pública de Chile que cursaban una misma asignatura, sobre Biología celular. Quienes participaron fueron parte de un proceso de consentimiento informado, donde se explicaban los objetivos del estudio, las características de la participación requerida y se garantizaba la voluntariedad y el anonimato del proceso. Dicho consentimiento se certificaba mediante la firma de un formulario en el que se reiteraban todas las condiciones antes señaladas. Posteriormente, se aplicó presencialmente el cuestionario MSLQ impreso en una sesión de aproximadamente 15 minutos, en conjunto con otras escalas que eran parte de un estudio más amplio.

Los datos se analizaron mediante el paquete estadístico STATA/SE 11.0.

\section{Resultados}

\section{Análisis factorial}

Como primer paso del análisis de las propiedades psicométricas del MSLQ, se analizó la estructura factorial de éste realizando un 
Análisis Factorial Exploratorio, EFA, usando el método de extracción de factores del Análisis de Eje Principal, AEP, más adecuado para el procesamiento de escalas compuestas como el MSLQ (Hair, Anderson, Tatham, \& Black, 2005; Tabachnick \& Fidel, 2006).

Se evaluó la pertinencia del EFA para los 81 ítems del cuestionario en la muestra de 409 estudiantes, aplicando el estadístico de adecuación muestral de Kaiser-Meyer-Olkin (KMO), que fue de .87 , y la prueba de esfericidad de Bartlett, que resultó estadísticamente significativa $\chi^{2}(3240)=14150.17 ; p<.001$.

Dado que ambos análisis apoyaron la pertinencia de un EFA, se procedió a estimar el número de factores del MSLQ, haciendo uso complementariamente de dos criterios: el criterio de Kaiser-Guttman o de raíz latente, que es uno de los más empleados con estos propósitos, y el Análisis Paralelo de Horn, que constituiría una herramienta más adecuada para este fin (Buja \& Eyuboglu, 1992).

El primer criterio, de Kaiser-Guttman, apuntó a la presencia de diez factores con valores propios (eigenvalues) mayores a 1 con autovalores de $13.13,4.76,3.75,3.16,2.61,1.77,1.56,1.26,1.10$ y 1.01 , que explicarían el $83.31 \%$ de la varianza total de los ítems.

El segundo criterio, el Análisis Paralelo de Horn en base a 2.500 muestras aleatorias, indicó la presencia de diez factores, con valores propios $(13,13,4.76,3.75,3.16,2.61,1.77,1.56,1.26$, 1.10 y 1.01) por sobre los valores propios obtenidos en las muestras aleatorias $(1.22,1.15$, $1.07,1.05,1.03,1.02,0.90,0.88,0.87$ y 0.82 ).

A partir de la congruencia de ambos criterios en una solución de diez factores, se decidió evaluar la organización de los ítems en esta estructura a partir de los coeficientes de configuración de cada ítem en cada factor, calculados mediante AEP con rotación oblicua Oblimin directo.

Al hacerlo, se encontró que 10 ítems presentaban coeficientes de configuración o cargas factoriales bajo .30, que es el valor mínimo sugerido como umbral para considerar una carga factorial como significativa (Hair et al., 2005). Adicionalmente, el décimo factor presentaba solamente tres ítems con cargas significativas, siendo estas de $.379, .361$ y -.320 .
En atención a estos problemas, se decidió eliminar el ítem 74 ("Aún cuando los materiales del curso sean aburridos y poco interesantes me las arreglo para mantenerme trabajando hasta que termino") que era el que presentaba las menores carga factorial (entre -.077 y .240), y repetir el análisis con los 80 ítems restantes.

Con este conjunto, se volvieron a calcular el estadístico KMO, que fue de .87 , y la prueba de esfericidad de Bartlett, que resultó estadísticamente significativa $\chi^{2}$ $(3160)=13935.30 ; p<.001$, ambos apoyando la pertinencia de un EFA.

En cuanto al número de factores, el primer criterio, de Kaiser-Guttman, apuntó a la presencia de nueve factores con valores propios (eigenvalues) mayores a 1 con autovalores de $12.96,4.76,3.70,3.14,2.61,1.77,1.55,1.26$, 1.09 , que explicarían el $81.49 \%$ de la varianza total de los ítems.

El segundo criterio, el Análisis Paralelo de Horn en base a 2.500 muestras aleatorias, indicó la presencia de nueve factores, con valores propios $(12.96,4.76,3.70,3.14,2.61,1.77,1.55$, $1.26,1.09)$ por sobre los valores propios obtenidos en las muestras aleatorias $(1.15,1.11,1.07,1.01$, $0.98,0.94,0.90,0.86$ y 0.81 ).

Dado que los criterios convergían en esta estructura, y que la eliminación del décimo factor identificado en el análisis anterior parecía esperable dadas las bajas saturaciones observadas en la matriz de configuración, se procedió a calcular la matriz de configuración con nueve factores. Esta vez, el noveno factor estaba compuesto por cinco ítems con cargas entre $.664 \mathrm{y}$ .353 , mostrando un funcionamiento aceptable. Sin embargo, nuevamente 10 ítems presentaban coeficientes de configuración bajo 30 .

Por ello se decidió repetir todo el análisis eliminando al ítem con menores coeficientes de configuración, que era el ítem 54 ("Antes de estudiar nuevos contenidos en profundidad, por lo general le doy un vistazo para ver cómo se organizan"), con saturaciones entre -.187 у .238. Este mismo procedimiento debió repetirse eliminando, consecutivamente los ítems: 56 ("Trato de cambiar la forma en que estudio para cumplir con los requerimientos y el estilo de enseñanza del profesor"), con saturaciones entre .044 y .252; 48 ("Me esfuerzo mucho para tener 
éxito en este curso aun cuando no me guste lo que estamos viendo"), con saturaciones entre -.213 y $.259 ; 30$ ("Quiero que me vaya bien en este curso porque para mí es importante demostrar mis habilidades a mi familia, mis amigos, empleadores u otros"), con saturaciones entre -.160 y $.260 ; 8$ ("Cuando doy una prueba pienso en las preguntas de otras partes de la misma que no puedo contestar"), con saturaciones entre -.129 у .262; 21 ("Espero que me vaya bien en este curso"), con saturaciones entre -.017 y .273 ; y 44 ("Si el material de apoyo es de difícil de entender, cambio la manera que tengo de leerlo"), que presentaba un coeficiente de entre -.078 y .287.

Al procesar los 73 ítems restantes, se volvieron a calcular el estadístico KMO, que fue de .86, y la prueba de esfericidad de Bartlett, que resultó estadísticamente significativa $\chi^{2}(2628)=12556.87 ; p<.001$, ambos apoyando la pertinencia de un EFA.

En cuanto al número de factores, el primer criterio, de Kaiser-Guttman, apuntó a la presencia de nueve factores con valores propios (eigenvalues) mayores a 1 con autovalores de $11.78,4.60,3.56,3.09,2.50,1.70,1.49,1.18$ у 1.06 que explicarían el $85.07 \%$ de la varianza total de los ítems.

El segundo criterio, el Análisis Paralelo de Horn en base a 2500 muestras aleatorias, indicó la presencia de nueve factores, con valores propios $(11.78,4.60,3.56,3.09,2.50,1.70,1.49,1.18$ y 1.06) por sobre los valores propios obtenidos en las muestras aleatorias $(1.10,1.02,0.95,0.92$, $0.89,0.86,0.80,0.75$ y 0.72 ).

Dado que nuevamente convergieron en una estructura de nueve factores, se obtuvo la matriz de configuración de esta estructura, encontrándose que en esta ocasión y con 73 ítems, todos éstos presentaban cargas sobre el umbral de .30 (Anexo $1)$.

A partir de los estos resultados, se procedió a la asignación de los ítems a los factores en que presentaban cargas estadísticamente significativas y la interpretación de los mismos.

A continuación se muestra la constitución final de los nueves factores, los que serán comparados con la estructura hipotética propuesta por Pintrich et al. (1991).

Factor I. Contiene los ítems 27, 17, 26, 23, $10,4,22,7$ y 24 (ordenados de mayor a menor coeficiente de configuración), tiene relación con el valor hacia las tareas o actividades de la asignatura por lo cual se mantiene el nombre de Valor de la tarea.

Factor II. Incluye los ítems 63, 67, 32, 39, $49,72,42,55$ y 79 , en conjunto se relacionan con la forma de estructurar y retener la información, se denominó Organización.

Factor III. Conformado por los ítems 15, 18, $20,13,6,12,29,9,25,1,2,31,16$ y 5 . Se asigna el nombre de Expectativas dado que representa casi en su totalidad a los ítems organizados originalmente en esta componente.

Factor IV. Contiene los ítems 76, 69, 64, 60, $73,80,69,37,35,46$ y 57 . En conjunto se orientan hacia la supervisión del aprendizaje, el esfuerzo y los recursos que el estudiante debe movilizar para ser efectivo, se denominó Autorregulación.

Factor V. Incluye los ítems 47, 71, 51, 62, 66, 70,36 y 38 , en conjunto hacen referencia al pensamiento crítico denominándose de esa forma.

Factor VI. Contiene los ítems 78, 81, 34, 61, 58,53 y 41, apuntan hacia un estudiante activo, comprometido con su aprendizaje, gestiona recursos internos y externos que le permiten alcanzar sus metas por lo cual se denominó Aprendizaje estratégico.

Factor VII. Conformado por los ítems 52, 43, 33, 77 y 65, hace referencia al Manejo del tiempo $\mathrm{y}$ ambiente de estudio, manteniendo dicho nombre para este factor.

Factor VIII. Incluye los ítems 28, 19, 14, 3 y 11, representa casi en su totalidad a la subescala de Ansiedad (Pintrich et al, 1991) por lo cual se mantiene dicho nombre.

Factor IX. Contiene los ítems 68, 45, 75, 50 y 40 , se relaciona con identificar cuando y a quienes acudir por ayuda denominándose Búsqueda de ayuda.

\section{Análisis de consistencia interna}

Como segundo paso, se procedió a calcular la consistencia interna de los factores identificados, a través del coeficiente de confiabilidad alfa de Cronbach. Adicionalmente, se evaluó la capacidad discriminativa de los ítems, calculando el coeficiente de correlación entre cada ítem y el total corregido del factor al que pertenecía (total sin considerar el ítem en cuestión). En la Tabla 1 
Tabla 1. Coeficientes de confiabilidad alfa de Cronbach y capacidad discriminativa de los ítems de los factores del MSLQ

\begin{tabular}{lccc}
\hline & $\begin{array}{c}\text { Alfa de } \\
\text { Cronbach }\end{array}$ & \multicolumn{2}{c}{ Correlación ítem-total corregido } \\
& Mín (ítem) & Máx (ítem) \\
\hline F1. Valor de la tarea & .87 & .39 (ítem 24) & .78 (ítem 27) \\
F2. Organización & .79 & .42 (ítem 39) & .58 (ítem 63) \\
F3. Expectativas & .83 & .32 (ítem 25) & .63 (ítem 20) \\
F4. Autorregulación & .78 & .31 (ítem 57) & .55 (ítem 69) \\
F5. Pensamiento crítico & .73 & .20 (ítem 38) & .55 (ítem 47) \\
F6. Aprendizaje & .78 & .31 (ítem 53) & .67 (ítem 78) \\
estratégico & & & \\
F7. Manejo del tiempo y & .69 & .33 (ítem 65) & .54 (ítem 43) \\
ambiente de estudio & & .22 (ítem 11) & .59 (ítem 28) \\
F8. Ansiedad & .67 & .28 (ítem 40) & .52 (ítem 68) \\
F9. Búsqueda de ayuda & .64 & .13
\end{tabular}

se indican los coeficientes obtenidos para cada factor y el mínimo y máximo de los coeficientes de correlación entre el ítem y el total corregido calculado en cada uno de éstos.

Los resultados muestran que la confiabilidad de los factores del MSLQ fluctuó entre un alfa de .64 y un Alfa de .87, lo que mostraría confiabilidades utilizables en contexto de investigación. Por otro lado, las correlaciones ítem total corregido estuvieron en casi todos los casos sobre .30, un valor considerado aceptable. Las únicas excepciones fueron los ítems 11, 13 y 38, lo que apunta a la necesidad de revisar la pertinencia de su inclusión en estos factores.

Dado que las confiabilidades mostraron ser adecuadas, se procedió a calcular los puntajes totales de cada factor, a través de la sumatoria de los códigos asignados a las respuestas de cada uno de sus ítems. De esta forma se obtuvieron los resultados que se muestran en la Tabla 2.

Tabla 2. Estadísticos descriptivos de las puntuaciones obtenidas por los estudiantes en el

\begin{tabular}{lcccc}
\multicolumn{5}{c}{ MSLQ } \\
& M & D.E. & Mín & Máx \\
\hline F1. Valoración de la tarea & 54.96 & 7.56 & 16 & 63 \\
F2. Organización & 49.90 & 8.92 & 12 & 63 \\
F3. Expectativas & 79.73 & 10.28 & 38 & 98 \\
F4. Autorregulación & 61.11 & 10.38 & 14 & 77 \\
F5. Pensamiento crítico & 38.83 & 8.13 & 77 & 56 \\
F6. Aprendizaje estratégico & 33.07 & 8.87 & 10 & 49 \\
F7. Manejo del tiempo y & 21.19 & 6.57 & 5 & 35 \\
ambiente de estudio & & & & \\
F8. Ansiedad & 21.78 & 6.54 & 5 & 35 \\
F9. Búsqueda de ayuda & 24.15 & 5.95 & 5 & 35 \\
\hline
\end{tabular}

Posteriormente, al evaluar la correlación entre los nueve factores identificados, se encontró 30 correlaciones estadísticamente significativas entre los 36 cruces realizados, de las cuales sólo cuatro eran inversas (Tabla 3).

Destaca la correlación significativa del factor Aprendizaje estratégico con todos los demás factores en intensidades variables y de manera negativa con Ansiedad ( $r=-.10)$. La correlación más alta se observa entre Aprendizaje estratégico y Organización ( $r=.54)$.

Por otra parte la ansiedad se correlaciona de forma negativa con expectativas, aprendizaje estratégico y manejo del tiempo y ambiente de estudio.

\section{Discusión}

A nivel latinoamericano y en países de habla hispana en general existen investigaciones en las cuales se aborda el rol de las estrategias de aprendizaje y la motivación en el proceso de aprendizaje empleando para ello diversos instrumentos, algunos de creación personal o adaptaciones de otros instrumentos (Boza \& Toscano, 2012; Boza \& Méndez, 2013; Förster \& Rojas-Barahona, 2010; García-Ripa, SanchezGarcia, \& Risquez, 2016; Islas \& López, 2012; Martínez-Fernández, 2007; Muñoz, 2005; Ramírez \& Cortés, 2003; Stover, Uriel, Freiberg, \& Fernández, 2015). Sin embargo, en el contexto universitario uno de los instrumentos más utilizado y reconocido en este campo a nivel mundial es el Motivated Strategies Learning Questionnaire (MSLQ) de Pintrich et al. (1991). 
Tabla 3. Correlación entre las puntuaciones obtenidas por los estudiantes en el MSLQ

\begin{tabular}{|c|c|c|c|c|c|c|c|c|}
\hline & 1 & 2 & 3 & 4 & 5 & 6 & 7 & 8 \\
\hline 1. Valor de la tarea & - & & & & & & & \\
\hline 2. Organización & $.44 * * *$ & - & & & & & & \\
\hline 3. Expectativas & $.47 * * *$ & $.29 * * *$ & - & & & & & \\
\hline 4. Autorregulación & $.47 * * *$ & $.54 * * *$ & $.33 * * *$ & - & & & & \\
\hline $\begin{array}{l}\text { 5. Pensamiento } \\
\text { crítico }\end{array}$ & $.30 * * *$ & $.42 * * *$ & $.34 * * *$ & $.25 * * *$ & - & & & \\
\hline $\begin{array}{l}\text { 6. Aprendizaje } \\
\text { estratégico }\end{array}$ & $.36 * * *$ & $.33 * * *$ & $.20 * * *$ & $.28 * * *$ & $.27 * * *$ & - & & \\
\hline $\begin{array}{l}\text { 7. Manejo del } \\
\text { tiempo y } \\
\text { ambiente estudio }\end{array}$ & $.10^{*}$ & $.30 * * *$ & $.17 * * *$ & $.27 * * *$ & $.29 * * *$ & $-.17 * * *$ & - & \\
\hline 8. Ansiedad & .09 & .08 & $-.23 * * *$ & $-.10^{*}$ & .03 & .07 & $-.20 * * *$ & - \\
\hline $\begin{array}{l}\text { 9. Búsqueda de } \\
\text { ayuda }\end{array}$ & $.13^{*}$ & $.35 * * *$ & .03 & $.21 * * *$ & $.18 * * *$ & .03 & $.20 * * *$ & .03 \\
\hline
\end{tabular}

Escasos estudios se han centrado en la evaluación de las propiedades psicométrica del instrumento a nivel de habla hispana, entre ellos los trabajo realizados en Venezuela (Cardozo, 2008), España (Roces, Tourón, \& González, 1995; Martínez \& Galán, 2000), Argentina (Donolo, Chiecher, Paolini, \& Rinaudo, 2008), México (Ramírez, Canto-Rodríguez, Bueno, \& Echazarreta, 2013) y Colombia (Sabogal, Barraza, Hernández, \& Zapata, 2011), este último empleando la versión corta del instrumento. En Chile el trabajo reportado por Herrera (2014), utilizó la versión Argentina del MSLQe, adaptando algunos ítems y evaluando solamente su confiabilidad mediante la prueba de Alfa de Cronbach, sin hacer evaluaciones respecto de su validez. Lo anterior muestra una evidente necesidad de ampliar estudios de validación que permitan determinar su utilidad para evaluar la motivación y estrategias de aprendizaje en estudiantes universitarios.

Para esta muestra de estudiantes universitarios chilenos los resultados revelan la presencia de nueve factores que se pueden diferenciar conceptualmente en las dos dimensiones propuestas para el instrumento y en general se obtiene una estructura consistente con la propuesta teórica de Pintrich et al. (1991), aunque existen diferencias específicas en el análisis de los ítems. Es importante recordar que bajo la mirada sociocognitiva en la cual sostienen su teoría los autores, la motivación y las estrategias de aprendizaje pueden cambiar de un curso a otro, en tal sentido lo recomendado es utilizar el instrumento en el contexto de una asignatura. En nuestro estudio, aunque los alumnos pueden ser diversos debido que provienen de diferentes carreras, el curso dictado no lo es. Éste tiene las mismas características, exigencias y tipo de estrategias de enseñanza, lo cual nos entrega una mirada particular respecto de la motivación y estrategias de aprendizajes en un contexto pedagógico y socio cultural diferente en cual fue construido el instrumento.

Se obtienen tres factores que se estructuran con ítems de la escala de motivación propuesta por Pintrich et al. (1991), los cuales reproducen las componentes de expectativas, afecto $\mathrm{y}$ valoración, sin embargo en esta última no se obtuvieron las subescalas de Orientación intrínseca y extrínseca sino una redistribución de sus respectivos ítems en los otros tres factores. Estos resultados coinciden con lo reportado por Cardozo (2008) en estudiantes universitarios venezolanos, donde también se observa una redistribución de los ítems de las subescalas mencionadas. Resultado similar ocurre en el estudio de Martínez y Galán (2000), en estudiantes universitarios españoles en particular con la subescala de Orientación intrínseca.

Los otros seis factores se estructuran con ítems de la escala de estrategias de aprendizaje, sin embargo se observa una mayor dispersión respecto a la propuesta original de Pintrich et al. (1991). Se reproducen con claridad las subescalas de Organización, Pensamiento crítico, Búsqueda de ayuda y Aprendizaje por pares; parcialmente la 
subescala de Manejo del tiempo y ambiente de estudio y Regulación del esfuerzo. La subescala de autorregulación metacognitiva se diluye por completo, en cinco de los seis factores de estrategias de aprendizaje. Estos resultados se relacionan con lo reportado en otros estudios donde se analiza la consistencia de la versión en castellano del MSLQ (Cardozo, 2008; Martínez \& Galán, 2000; Roces et al., 1995) los cuales evidencian lo difícil de separar algunos elementos de gestión de recursos del componente cognitivo. Como señala Roces et al. (1995), lo anterior se puede deber a una falta de claridad en la determinación del constructo subyacente a cada una de las estrategias de aprendizaje que pretende medir o a las diferencias de contexto.

Llama la atención los factores cuatro y seis, que combinan ítems de las tres componentes de la escala de estrategias de aprendizaje. Estos factores apuntan hacia un estudiante con un aprendizaje más estratégico que combina estrategias cognitivas, metacognitivas y de gestión para alcanzar sus metas, aunque cada factor revela información diferente de cómo los estudiantes construyen sus aprendizajes. El factor cuatro involucra la gestión del tiempo, ambiente y esfuerzo pero movilizando estrategias de orden más simples, lo cual puede estar influenciado por las características de la asignatura, de nivel básico donde las exigencias de aprobación no demandan estrategias de orden superior, sino más bien de memorización y repetición de conocimientos.

Por otra parte, el factor seis se relaciona con la elaboración de aprendizaje apoyado por el trabajo con otros, ya sean sus pares o el docente. Esto es muy importante en el contexto de esta asignatura donde la mayor parte del curso se basa en trabajo individual, sin embargo estos resultados nos muestran que para apoyar el aprendizaje de cierto grupo de estudiantes se necesitan experiencias de trabajo colaborativo.

Si bien nuestros resultados no replican con exactitud las 15 subescalas propuestas para el instrumento los ítems se reorganizan en nueve factores que en su mayoría se mantienen dentro de los seis componentes que fueron planteados por Pintrich et al. (1991), observándose más bien una reorganización de los ítems lo cual es esperable debido a las diferencias de contexto en el cual está siendo evaluado el instrumento, con diferencias que van desde el lenguaje hasta los métodos de enseñanza-aprendizaje empleados a nivel universitario, y por que, hasta la fecha, no se ha podido encontrar una estructura replicable del MSLQ en las diferentes poblaciones en que se ha estudiado. Por otra parte la organización en estos nueve factores muestra niveles de confiabilidad aceptables en contexto de investigación lo que nos indica que el instrumento mide lo que pretende medir.

En respaldo de lo anterior, los mismo autores reportan que con el MSLQ, administrado a diferentes poblaciones como estudiantes de bachillerato o de universidad, emergen estructuras factoriales diferentes (Pintrich \& De Groot, 1990 ; Pintrich et. al 1991) pero los resultados caben dentro del modelo conceptual general y recomiendan que en futuras investigaciones se aborde si estas diferencias en el desarrollo de las estructuras de los factores son en función de la varianza del método o en realidad reflejan diferencias en el desarrollo de la motivación y la cognición (Duncan \& McKeachie, 2005).

Con base en estos resultados podemos obtener información valiosa de la forma en que nuestros estudiantes se involucran en el proceso de aprendizaje en el contexto de una asignatura en particular.

Sin embargo, quedan pendientes otras evaluaciones psicométricas del instrumento como su estabilidad temporal. Aunque, como señalan Duncan y McKeachie (2005) producto del amplio interés en el MSLQ en diferentes disciplinas y países también, hay que recordar las limitaciones de los instrumentos de autoinforme, en particular la fiabilidad en términos de estabilidad en el tiempo y contextos. Las medidas tradicionales de la estabilidad de aspecto fiabilidad son difíciles de usar para los instrumentos que tienen por objeto aprovechar construcciones que son dependientes del contexto.

En resumen nuestros resultados confirman que el MSLQ es un instrumento confiable de medición de la motivación y las estrategias de aprendizaje y aplicable en contexto de investigación. Sin embargo es necesario realizar nuevas investigaciones que permitan indagar respecto de la falta de consistencia con el modelo propuesto por Pintrich et al. (1991) en particular las relativas a la escala de estrategias de 
aprendizaje, buscando, por ejemplo realizar un análisis factorial confirmatorio del MSLQ en una muestra más amplia y heterogénea de estudiantes chilenos, a fin de identificar cuál de todas las soluciones identificadas para este cuestionario en español presenta un mejor ajuste empírico. Lo anterior permitiría avanzar hacia una definición más precisa de esta componente y del instrumento en general que permita evaluar de manera representativa a nuestro contexto local.

\section{Referencias}

Boza, A., \& Méndez, J. (2013). Aprendizaje motivado en alumnos universitarios: Validación y resultados generales de una escala. Revista de Investigación Educativa, 31, (2), 331-347. doi:10.6018/rie.31.2.163581

Boza, A., \& Toscano, M. (2012). Motivos, actitudes y estrategias de aprendizaje: Aprendizaje motivado en alumnos universitarios. Profesorado. Revista de Currículum y Formación de Profesorado, 16 (1), 125-142. doi:10.6018/rie.31.2.163581

Buja, A., \& Eyuboglu, N. (1992). Remarks on parallel analysis. Multivariate Behavioral Research, 27, 509-540. doi: 10.1207/s15327906mbr2704_2

Cardozo, A. (2008). Motivación, aprendizaje y rendimiento académico en estudiantes del primer año universitario. Laurus, 14 (28), 209-237. http://www.redalyc.org/articulo.oa?i $\mathrm{d}=76111716011$

Duncan, T. G., \& McKeachie, W. J. (2005). The making of the motivated strategies for Learning Questionnaire. Educational Psychologist, 40(2), 117-128. doi: 10.1207/s15326985ep4002_6

Credé, M., \& Phillips, A. (2011). A meta-analytic review of the Motivated Strategies for Learning Questionnaire. Learning and Individual Differences 21, 337-346. doi: 10.1016/j.lindif.2011.03.002

Díaz, C. (2008). Modelo conceptual para la deserción estudiantil universitaria chilena. Estudios pedagógicos (Valdivia), 34(2), 6586. doi: 10.4067/S0718-07052008000200004

Donolo, D., Chiecher, A., Paolini, P., \& Rinaudo, M. C. (2008). MSLQe-MSLQvv. Motivated strategies learning questionnaire. Propuestas para la medición de la motivación y el uso de estrategias de aprendizaje. Río Cuarto, Argentina: Universidad Nacional de Río Cuarto.

Förster, C., \& Rojas-Barahona, C. (2010). Adaptación y Validación del Cuestionario de Rasgos de Pensamiento de O’Neil y colaboradores: Metacognición y motivación en la solución de problemas. Revista Iberoamericana de Diagnóstico y Evaluación - e Avaliação Psicológica. RIDEP, 30 (2), 934.

Garcia-Ripa, M., Sanchez-Garcia, M., \& Risquez, A. (2016). Estrategias de Aprendizaje y Autorregulación Motivacional. Identificación de Perfiles para la Orientación de Estudiantes Universitarios de Nuevo Ingreso. Revista Iberoamericana de Diagnóstico y Evaluación - e Avaliação Psicológica. RIDEP, 41 (1), 3957.

Hair, J., Black, W., Babin, B., Anderson, R., \& Tatham, R. (2005). Multivariate data analysis. (6th ed.). New Jersey: Prentice Hall.

Herrera, F. (2014). Motivación, estrategias de aprendizaje y rendimiento académico, en alumnos de Nutrición y Dietética de la Universidad del Desarrollo. Rev Educ Cienc Salud, 11 (1), 38-46.

Islas, M., \& López, M. (2012). Evaluación de estrategias de aprendizaje y habilidad verbal en una muestra de estudiantes universitarios. Enseñanza e Investigación en Psicología, 17 (1), 117-135.

Khatib, A. (2010). Meta-cognitive self-regulated learning and motivational beliefs as predictors of college students' performance. International Journal for Research in Education, 27, 57-72.

Kitsantas, A., Winsler, A., \& Huie, F. (2008). Self-regulation and ability predictors of academic success during college: A predictive validity study. Journal of Advanced Academics, 20(1), 42-68. doi: 10.4219/jaa2008-867

Martínez-Fernández, R. (2007). Concepción de aprendizaje y estrategias metacognitivas en estudiantes universitarios de psicología. Anales de psicología, 23 (1), 7-16.

Martínez, R., \& Galán, F. (2000). Estrategias de aprendizaje, motivación y rendimiento 
académico en alumnos universitarios. REOP, 11 (19), 35-50. doi 10.5944/reop.vol.11.num.1 9.2000 .11323

Martínez, R., Hernández, M. J., \& Hernández, M. V. (2006). Psicometría. Madrid: Alianza.

Muñoz, M. (2005). Estrategias de aprendizaje en estudiantes universitarias. Revista Electrónica de Psicología Científica.com. Disponible en línea: http://www.psicologiacientifica.com/bv/ psicologia-62-1-estrategias-de-aprendizaje-enestudiantes-universitarias.html.

Núñez, J. (2009). Motivación, aprendizaje y rendimiento académico. Actas do $\mathrm{X}$ Congresso Internacional Galego Português de Psicopedagogia. (pp. 41-64). Braga: Universidade do Minho.

Pintrich, P. (1986). Motivation and learning strategies interactions with achievements. Presented at the American Educational Research Association Convention. San Francisco, CA, EE. UU.

Pintrich, P. (1989). The dynamic interplay of student motivation and cognition in the college classroom in M. Maehr \& C. Aimes (Eds.). Advances in motivation and achievement: Motivation enhancing environments 6, 117-160. Greenwich, CT: Jai Press, Inc.

Pintrich, P., \& De Groot, E. (1990). Motivational and self-regulated learning components of classroom academic performance. Journal of Educational Psychology, 82(1), 33-40.

Pintrich, P., \& Garcia, T. (1991). Student goal orientation and self-regulation in the college classroom. In M.L. Maehr \& P.R. Pintrich (Eds.), Advances in motivation and achievement: Goals and self-regulatory processes, 7, pp. 371-402. Greenwich, CT: JAI Press.

Pintrich, P., Smith, D., García, T., \& Mckeachie, W. (1991). A manual for the use of the motivational strategies for learning questionnaire (MSLQ). AnnArbor, MI: NCRIPTAL, the University of Michigan.

Pintrich, P., Smith D., Garcia, T., \& McKeachie, W. (1993). Reliability and predictive validity of the motivated strategies for learning questionnaire (MSLQ). Educational and Psychological Measurement, 53, 801-803. Doi: 10.1177/0013164493053003024
Ramírez, M., Canto y Rodríguez, J., Bueno, J., \& Echazarreta, A. (2013). Validación psicométrica del motivated strategies for Learning Questionnaire en Universitarios Mexicanos. Electronic Journal of Research in Educational Psychology, 11(1), 193-214.

Ramírez, P., \& Cortés, P. (2003). Motivación y rendimiento en una carrera del área de química de la Universidad católica del norte Chile. Psicoperspectivas, 2 (1), 129-155.

Rinaudo, M., Chiecher, A., \& Donolo, D. (2003). Motivación y uso de estrategias en estudiantes universitarios. Su evaluación a partir del Motivated Strategies Learning Questionnaire. Anales de Psicología, 19 (1), 107-119.

Rinaudo, M., De la Barrera, M., \& Danolo, D. (2006). Motivación para el aprendizaje en alumnos universitarios. Revista Electrónica de Motivación y Emoción, 9 (22), 1-19.

Roces, C., González-Pienda, J., Núñez, J., González-Pumariega, S., García, M., \&. Alvarez, L. (1999). Relaciones entre motivación, estrategias de aprendizaje y rendimiento académico en estudiantes universitarios. Mente y Conducta en situación educativa, I (1), 41-50.

Roces, C., Tourón, J., \& González, M. (1995). Validación preliminar del CEAM II (cuestionario de estrategias de aprendizaje y motivación II). Psicológica, 16, 347- 366.

Sabogal. L., Barraza, E., Hernández, A. \& Zapata, L. (2011). Validación del cuestionario de motivación y estrategias de aprendizaje forma corta -mslq sf, en estudiantes universitarios de una institución pública-santa marta. Psicogente, 14 (25), 36-50.

Sachs, J., Law, Y., Chan, C., \& Rao, N. (2001). A nonparametric itema analysis of the motivated strategies for learning questionnaire-Chinese version. Psychologia, 44, 197-208. doi: 10.2117/psysoc.2001.197

Saks, K., Leijen, A., Edovald, T., \& Õun, K. (2015). Cross-cultural adaptation and psychometric properties of the estonian Version Of MSLQ. Procedia - Social and Behavioral Sciences, 191, 597-604. doi: 10.1016/j.sbspro.2015.04.278

Servicio de Información de Educación Superior (SIES). (2015). Informe Matrícula 2015 de Educación Superior. http://www.mifuturo.cl/ 
images/Informes_sies/Matricula/matricula_20

15_principales_resultados.pdf

Stover, J., Uriel, F., Freiberg, A., \& Fernández, M. (2015). Estrategias de aprendizaje y motivación académica en estudiantes universitarios de Buenos Aires. Psicodebate, 15 (1), 69-92.

Tabachnick, B., \& Fidell, L. (2006). Using multivariate statistics. (5th ed). Boston, Massachusetts: Allyn \& Bacon. 


\section{Anexo 1}

Matriz de configuración del MSLQ para estudiantes universitarios obtenida mediante Análisis de Eje Principal con rotación Oblimín

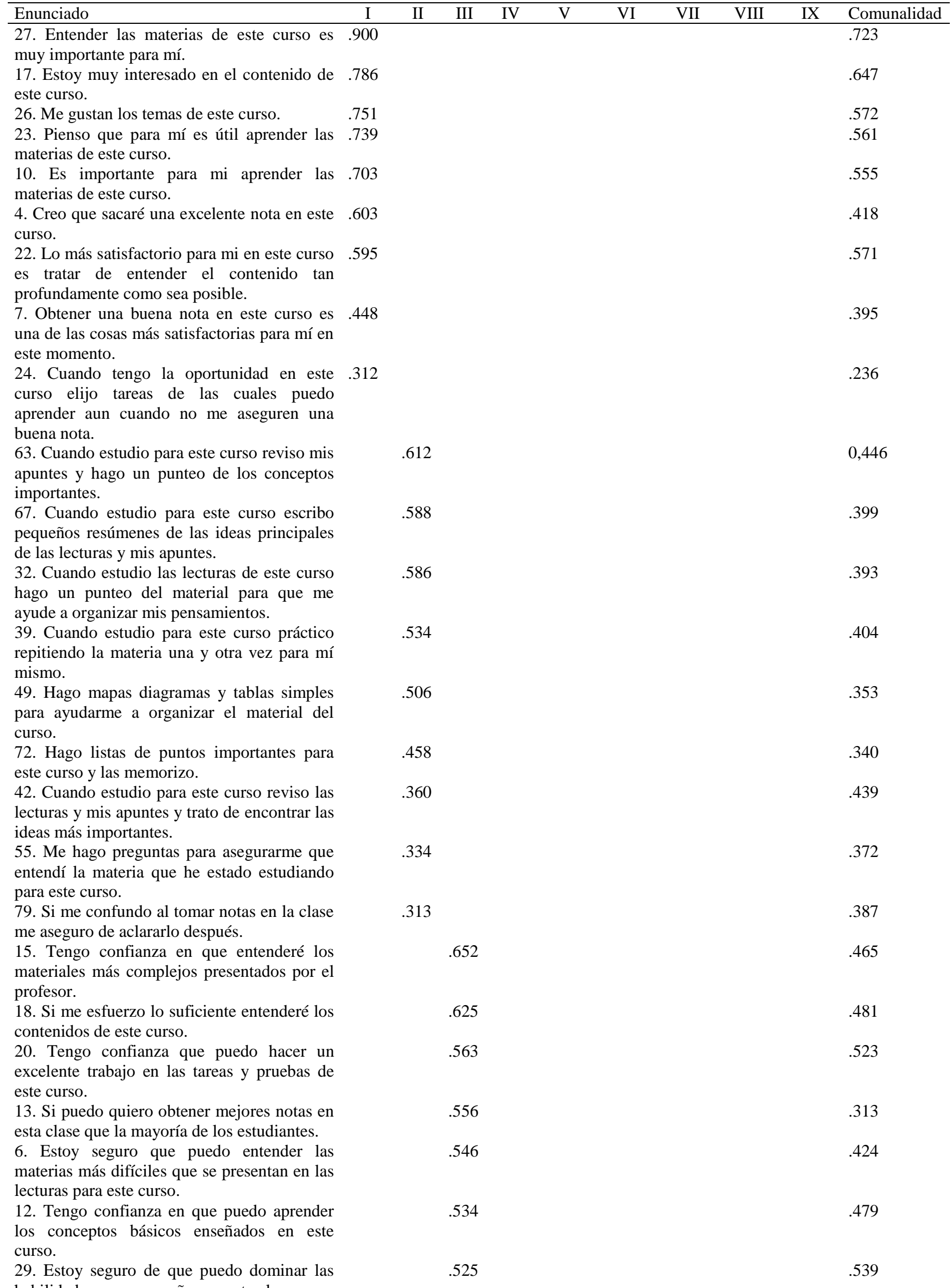


9. Si no aprendo las materias de este curso es

mi propia responsabilidad.

25. Si no entiendo la materia de este curso es

porque no intento lo suficiente.

1. En este curso prefiero materiales que me

cosas.

2. Si estudio en la forma apropiada podré aprender el material o contenido de esta asignatura Cuando doy una prueba pienso en lo mal que me está yendo comparado con mis compañeros.

31. Considerando la dificultad de este curso el (la) profesor(a) y mis habilidades pienso que me irá bien.

16. En un curso como este prefiero materiales que estimulen mi curiosidad aun cuando sean difíciles de aprender.

5. Creo que sacaré una excelente nota en este curso.

76. Cuando estudio para este curso trato de ver cuáles conceptos no entiendo bien.

69. Trato de entender el material de este curso conectando lecturas y conceptos de las clases.

64. Cuando leo para este curso trato de relacionar el material a lo que ya sé.

60. Cuando el trabajo de este curso es difícil me doy por vencido o solo estudio las partes fáciles.

73. Asisto regularmente a las clases.

80. Raramente tengo tiempo para revisar mis apuntes y lecturas antes de una prueba.

37. A menudo me siento tan aburrido o flojo cuando estudio para esta asignatura que abandono antes de terminar lo que tenía planeado hacer.

35. Generalmente cuando estudio para este curso lo hago en un lugar donde me pueda concentrar.

46. Cuando estudio para este curso leo mis apuntes y lecturas una y otra vez.

57. Encuentro de que a menudo he estado leyendo para este curso pero no tengo idea de que se trata.

47. Cuando se presenta una teoría interpretación o conclusión en la clase o en las lecturas trato de decidir si hay buena evidencia que las apoye.

71. Cada vez que leo o escucho una aseveración o conclusión en esta clase pienso en posibles alternativas.

51. Trato el material del curso como un punto de partida he intento desarrollar mis propias ideas al respecto.

62. Trato de relacionar las ideas de este tema con las de otros cursos cada vez que sea posible.

66. Trato de jugar con ideas propias relacionadas con lo que estoy aprendiendo en este curso.

70. Me aseguro de mantenerme al día con las lecturas y tareas semanales de este curso.

36. Cuando leo para este curso invento preguntas que me ayuden a enfocarme en la lectura.

38. A menudo me encuentro cuestionando cosas que escucho o leo en este curso para decidir si las encuentro convincentes.

78. Cuando estudio para este curso me 
establezco metas para orientar mis actividades en cada periodo de estudio.

81. Trato de aplicar ideas obtenidas de las

ecturas del curso en otras actividades de clases como actividades grupales $y$ discusiones.

34. Cuando estudio para este curso a menudo trato de explicar la materia a compañeros o amigos.

61. Cuando estoy estudiando para este curso en vez de simplemente leer un tema trato de pensar en el y decidir que se supone que debo aprender.

58. Le pido al profesor que clarifique conceptos que yo no entiendo bien.

53. Cuando estudio para este curso junto información de distintas fuentes tales como clases lecturas y discusiones.

41. Cuando me confundo acerca de algo que estoy leyendo para este curso vuelvo atrás y trato de aclararlo.

52. Encuentro difícil apegarme a un horario de estudio.

43. Hago un buen uso de mi tiempo de estudio para este curso.

33. Durante la clase a menudo pierdo información importante por estar pensando en otras cosas.

77. A menudo encuentro que no invierto mucho tiempo en este curso debido a otras actividades.

65. Tengo un lugar reservado para estudiar.

28. Siento que mi corazón se acelera cuando doy una prueba.

19. Tengo una sensación de ansiedad y malestar cuando doy una prueba.

14. Cuando doy una prueba pienso en las consecuencias de que me vaya mal.

3. Pienso que lo que aprendí en este curso me será útil para otros cursos.

11. Lo más importante para mí ahora es subir mi promedio general de manera que mi principal interés en este curso es obtener una buena nota.

68. Cuando no puedo entender el material de este curso le pido ayuda a otro estudiante de este curso.

45. Trato de trabajar con otros estudiantes de

este curso para hacer las tareas.

75. Trato de identificar a los estudiantes de este curso a los cuales puedo pedir ayuda de ser necesario.

50. Cuando estudio para este curso a menudo programo tiempo para discutir la materia con un grupo de estudiantes de la clase.

40. Aun cuando tenga dificultades aprendiendo las materias de este curso trato 